\title{
Exposure to Urine of Canids and Felids, but not of Herbivores, Induces Defensive Behavior in Laboratory Rats
}

\author{
Markus Fendt
}

Received: 1 February 2006 /Revised: 7 August 2006 / Accepted: 9 August 2006 /

Published online: 8 November 2006

(C) Springer Science + Business Media, Inc. 2006

\begin{abstract}
Predator odors induce defensive behavior in many prey species. For various reasons, studies carried out up to now have been unable to establish whether predator odor recognition is innate or not. Mostly, only particular odors or wild-living (i.e., experienced) test animals have been used in these studies, restricting the conclusiveness of the observations. In the present study, the behavioral effects of exposure to different predator odors on predator odor-naive laboratory male rats were compared with the effects of different nonpredator odors and of a no-odor control stimulus. Results show that exposure to urine of canids and felids, but not of herbivores or conspecifics, induce defensive behaviors. Taken together, the study provides support for the hypothesis that there is an innate recognition of predator odors in laboratory rats.
\end{abstract}

Keywords Defensive behavior - Innate fear - Predator odor - Predator-prey interaction - Rat . Rattus norvegicus $\cdot$ Urine

\section{Introduction}

Carnivorous mammals such as wolves, foxes, bobcats, cougars, coyotes, and cats are important predators for small rodents such as mice and rats (e.g., Glowacinski and Profus, 1997; Goldyn et al., 2003). These carnivorous mammals are usually territorial and use urine and feces to mark their territory (e.g., Gese and Ruff, 1997). This scent marking has the advantage that possible competitors may be deterred, but also has the disadvantage that prey animals could be warned by the scent marks. Indeed, several field studies have shown that prey species avoid areas that are marked by predators or show an increase in defensive behaviors, e.g., risk assessment behavior (Dickman and Doncaster, 1984; Dickman, 1992;

\footnotetext{
M. Fendt $(\bowtie)$

Tierphysiologie, Zoologisches Institut, Fakultät für Biologie, Universität Tübingen, Auf der Morgenstelle 28, 72076 Tübingen, Germany

e-mail: markus.fendt@novartis.com

Present Address:

M. Fendt

Novartis Institutes for Biomedical Research, Neuroscience Research,

WSJ-386.3.44, Lichtstrasse 35, 4056 Basel, Switzerland
} 
Stapley, 2003; Labra and Niemeyer, 2004; Amo et al., 2004; reviewed in Kats and Dill, 1998; Apfelbach et al., 2005). Since prey species also respond to unknown predator odors, some have suggested that recognition of predator odors as a sign of potential danger is innate (Epple et al., 1993; Apfelbach et al., 2005). Such an innate recognition of predator odor would be an enormous advantage. After perceiving a predator odor, the prey animal can avoid the encounter with the predator. This is a highly critical advantage because the very first encounter with a predator usually ends fatally for the prey (Edut and Eilam, 2003). However, another possible explanation for the observed effects of unknown predator odors is that they contain components of a known predator. This would mean that recognition of the unknown predator odor is not innate but is rather based on previous experience with other predators.

A better way to test for innate recognition of predator odors is to use prey animals that have had no previous experience of predator odors, i.e., laboratory-reared animals, and to observe their behavior during exposure to different predator and nonpredator odors. A number of studies have shown that the odors of certain predators (usually cats or foxes) induce autonomic and behavioral signs of fear in laboratory animals (reviewed in Blanchard et al., 1990a; Dielenberg and McGregor, 2001; Apfelbach et al., 2005; Fendt et al., 2005; Takahashi et al., 2005). Since these laboratory animals (rats, mice) were totally naive to predators and predator odors, this is evidence that rats and mice innately recognize predator odors as signs of potential danger. Major drawbacks to these studies, however, are that in each case they have invariably used only one particular predator odor and that they have carried out control experiments either with synthetic odors instead of nonpredator odors or simply by eliminating all odors. As a result, the responses observed in these studies were perhaps general responses to unfamiliar animal odors.

The aim of the present study was to collect further data supporting the hypothesis that predator odor recognition is innate. To this end, the behavioral effects of different rodent odors were tested on male laboratory rats that were all naive to the odors used in the study. Urine samples from different canids and felids (wolf, coyote, bobcat, fox, and cat) were used as test stimuli, and urine samples of herbivore nonpredators (cow and horse) or of female rats were used as control stimuli, along with saline (i.e., no odor). Each rat was exposed in a test arena to all odors in a randomized order, and the behavior of the rat was observed. If laboratory rats are innately able to recognize predator odors, defensive behavior is expected during exposure to predator urine, but not during exposure to the control stimuli. The following defensive behaviors of rats are described in laboratory but also in field studies (Blanchard and Blanchard, 1971, 1990; Dielenberg and McGregor, 2001; Carobrez et al., 2001; McNaughton and Corr, 2004; Apfelbach et al., 2005): flight, hide and avoidance behavior, freezing behavior, potentiation of defensive reflexes, and "risk assessment behavior" such as flat back approaches, prolonged approach to and short direct contacts with the potentially dangerous stimuli.

\section{Methods and Materials}

\section{Animals}

Fourteen male Sprague-Dawley rats (Charles River GmbH, Sulzfeld, Germany) weighing 220-260 g (age, 3-4 mo) at the beginning of the experiments were used. Animals were maintained on a 12:12 hr light/dark cycle, and food and water were available ad libitum. 
All experiments were performed in accordance with ethical guidelines for the use of animals in experiments and were approved by the local animal care committee (Regierungspräsidium Tübingen, ZP 4/02).

\section{Odor Probes}

Urine probes of bobcats, cougars, foxes, coyotes, and wolves were purchased from PredatorPee Inc. (Bangor, ME, USA). Urine probes of female cows and horses (females, males, geldings) were self-collected at two ecological farms in Waldhausen, a village close to Tübingen. Urine probes of female rats $(N=6)$ were self-collected by using a metabolic cage at the animal facility of the Department of Animal Physiology of the University of Tübingen. Lastly, urine probes of healthy female and male cats were drawn by a local veterinarian by means of bladder catheterization. All samples were collected in the week before the experiments and were stored in a refrigerator at $-18^{\circ} \mathrm{C}$ until they were used.

Samples were presented as follows: $3 \mathrm{ml}$ of predator or nonpredator urine or saline (control) were administered in a Petri dish $(5 \mathrm{~cm}$ diam.). The Petri dish was put in one corner of the arena (see below) at a distance of $5 \mathrm{~cm}$ from each wall of the arena.

\section{Apparatus}

All behavioral tests were conducted in a square arena with walls and a cover made from transparent Plexiglass $(92 \times 92 \mathrm{~cm}$; ActiMot, TSE Systems, Bad Homburg, Germany). Illumination of the arena was between 200 and $250 \mathrm{~lx}$. There was no ventilation of the arena during the tests. Rat movements were monitored by infrared detectors (distance between two detectors: $2.5 \mathrm{~cm}$, height: $2.5 \mathrm{~cm}$ ), and rearings were detected by a second arrangement of infrared detectors (height: $12.5 \mathrm{~cm}$ ). The ActiMot software automatically calculated the motor activity [distance traveled $(\mathrm{m})$ ], the number of rearings, the duration of "resting behavior" (activity $<1 \mathrm{~cm} / \mathrm{sec}$ ), the total time spent in the middle of the arena (size of the middle: $46 \times 46 \mathrm{~cm}$ ), and the total time spent in the corner with the urine probe (size of the "corner": $23 \times 23 \mathrm{~cm})$.

In addition, the behavior of the animal was videotaped. The videotapes were analyzed by an observer who was unaware of the test conditions. The duration and frequency of the following types of behavior were recorded: flat back approach, contact with the sample, grooming, and freezing. The time elapsed before first contact with the sample and the number of fecal boli were recorded.

\section{Behavioral Procedures}

To familiarize the rats with the arena, each animal was placed together with odorless saline solution in a Petri dish ( $3 \mathrm{ml}$ saline) into the arena for $10 \mathrm{~min}$ once per day on 3 consecutive d. On the following $10 \mathrm{~d}$, a urine sample or saline control solution was put in one corner of the arena, and the rat was put in the opposite corner. The rat's behavior was monitored for the next $10 \mathrm{~min}$ by the infrared detectors and videotaped as described above.

Each rat was tested daily between 8:00 AM and 4:00 PM with one of the samples. Samples were presented in a pseudorandomized order, and the corner with the urine samples (and thereby also the animal's "start" corner) was changed in pseudorandomized order. The arena was cleaned with soapy water and $70 \%$ ethanol after each test, and then ventilated with clean air for $45 \mathrm{~min}$ (ca. $15 \mathrm{l} / \mathrm{min}$ ). Control studies in our laboratory with 
trimethylthiazoline, an intensive synthetic predator odor inducing strong fear behavior, showed that the cleaning procedure removed all odors that might affect the animal's behavior.

\section{Statistical Analysis}

Data are presented as mean values with standard error of the mean. The data were also grouped for each animal according to sample type (i.e., predator urine, nonpredator urine, female rat urine, and saline controls). All data were normally distributed except for the number of rearings. Normally distributed data were analyzed by repeated-measures analyses of variance (ANOVAs) with odor or odor group as a within-subject factor. Student's $t$-test (with Bonferroni correction) was used for pairwise comparisons with the saline control. Nonnormally distributed data were analyzed by a Kruskal-Wallis test; pairwise comparisons were done by the nonparametric multiple comparison according to Zar (1999, p. 224).

\section{Results}

The type of urine used strongly affected the time the animals spent in the corner with the urine probe (ANOVA: $F_{9,108}=11.59, P<0.001$; Figure 1a). Pairwise comparisons with the saline control showed that animals spent less time in the sample corner in the case of urine from wolves $(t=5.01, P<0.001)$, coyotes $(t=4.7, P=0.001)$, bobcats $(t=3.75, P=0.003)$, cougars $(t=3.79, P=0.003)$, and foxes $(t=2.42, P=0.03)$, but more time in this corner in the case of urine from female rats $(t=2.66, P=0.02)$. The urine of cows, horses, and cats did not affect the time spent in the sample corner $(t<1.09, P>0.30)$. After the data had been grouped according to sample type, ANOVA revealed significant effects of the different sample types $\left(F_{3,39}=14.78, P<0.001\right)$. Pairwise comparison with the saline controls showed significant effects for predator urine $(t=2.6, P=0.02)$ and female rat urine $(t=3.04, P=$ $0.009)$, but not for nonpredator urine $(t=1.21, P=0.25)$. Furthermore, the effects of exposure to predator urine and nonpredator urine differed $(t=5.26, P<0.001)$. Typical traces of an individual rat during exposure to different urine samples illustrate avoidance behavior indicated by reduced time spent in the corner with the predator urine samples (Figure 2).

Flat back approaches are not direct and fast approaches to the samples; rather, they are more of a hesitant, sneaking approach to the threatening stimulus, with many stops. Nonparametric analysis with the Kruskal-Wallis test revealed an effect of urine samples on the number of flat back approaches $(H=29.87, P<0.001$; Figure 1b). Post-hoc pairwise comparisons showed an increase in the number of flat back approaches during exposure to the urine of coyotes, bobcats, cougars, foxes, and wolves $(Q>10.56, P<0.001)$, but not during exposure to the urine of cats, cows, horses, and female rats $(Q=0)$. The results were confirmed by an analysis of the grouped data $(H=19.72, P<0.001)$ : compared with the saline controls, predator urine led to an increase in the number of flat back approaches $(Q=$ 6.35, $P<0.001)$, whereas the urine of nonpredators or female rats did not $(Q=0)$. In addition, a difference was found between the effects of predator urine and nonpredator urine $(Q=56, P=0.007)$.

The type of urine used influenced the time elapsed before the animal's initial contact with the samples (ANOVA: $F_{9,108}=2.41, P<0.02$; Figure $1 \mathrm{c}$ ). On the other hand, pairwise comparisons showed no significant differences in this elapsed time before contact with the saline sample and other samples $(t<2.1)$. However, after the data were grouped (ANOVA: 


\section{a}
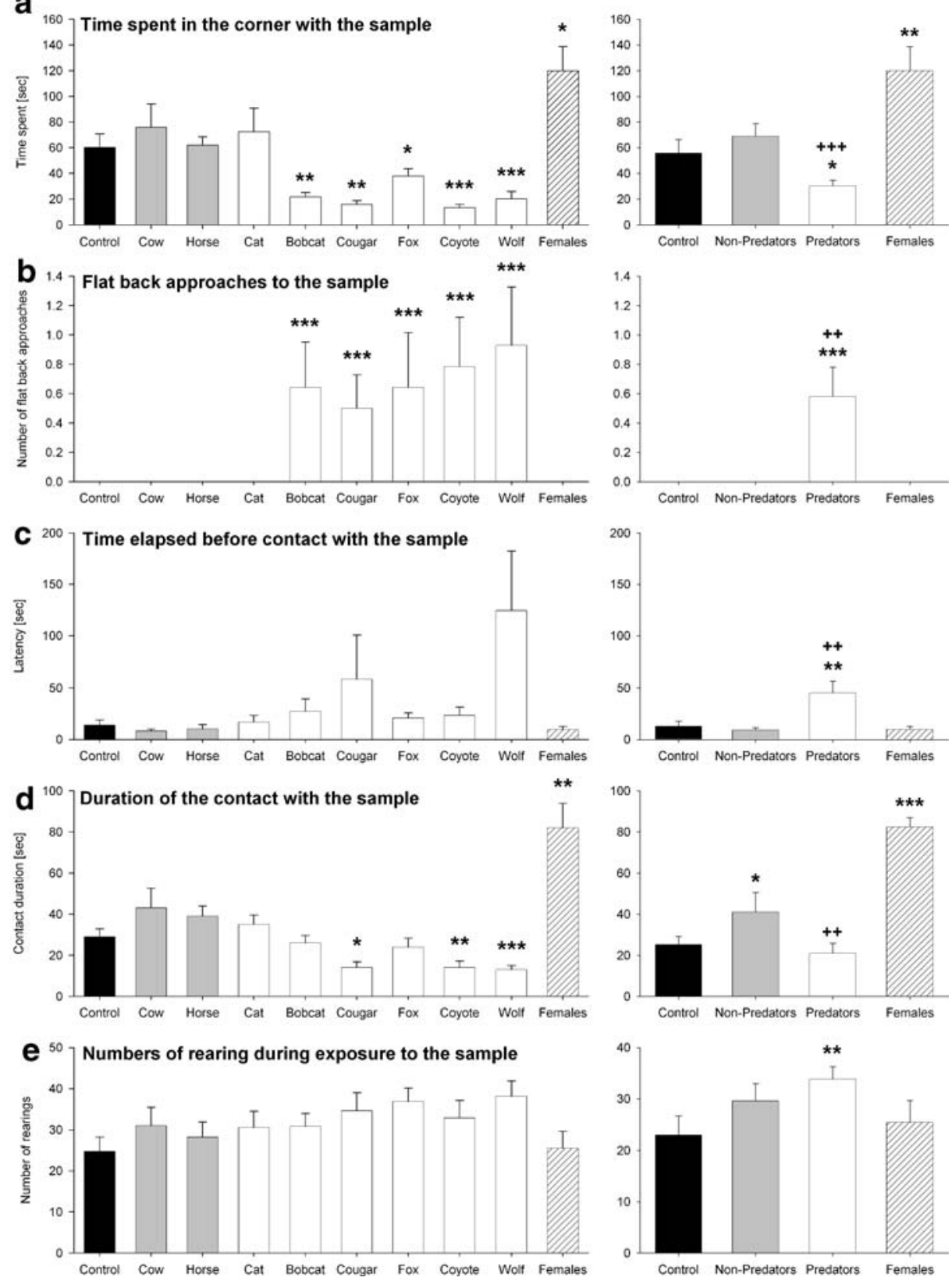

Fig. 1 Behavioral effects of exposure to different urine samples. (a) Time spent in the corner with the sample; (b) numbers of flat back approaches to the sample; (c) time elapsed before contact with the sample; (d) duration of the contact with the sample; (e) numbers of rearing during exposure to the different samples. Left panel illustrates the mean behavioral effects to each urine sample $( \pm \mathrm{SE})$; right panel illustrates the mean behavioral effects to the different odor groups $( \pm \mathrm{SE}) .{ }^{* * *} P<0.001,{ }^{* *} P<0.01,{ }^{*} P<0.05$, comparison with the saline control (after an ANOVA). ${ }^{+++} P<0.001,{ }^{++} P<0.01$, comparison with exposure to nonpredator urine (after an ANOVA) 
Fig. 2 Typical examples of traces of one rat during exposure to the saline control (a), to urine of a female rat (b), to urine of the nonpredator cow (c) and horse (d), and to urine of the predator cougar (e) and coyote (f). Dark ring represents the placement of the urine sample
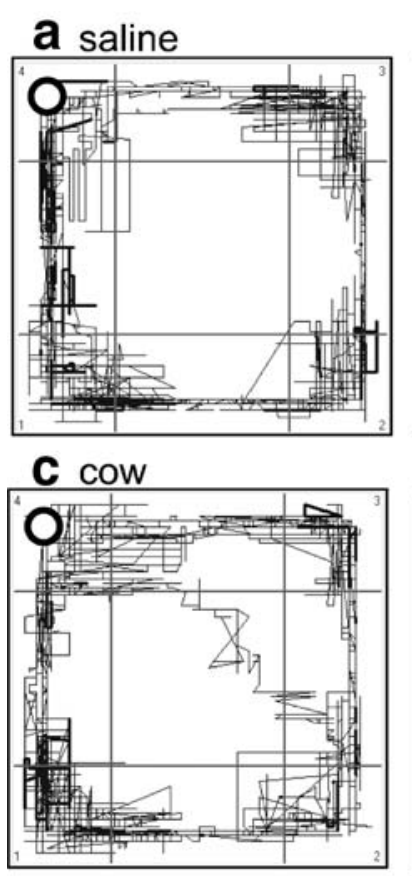

b female rat
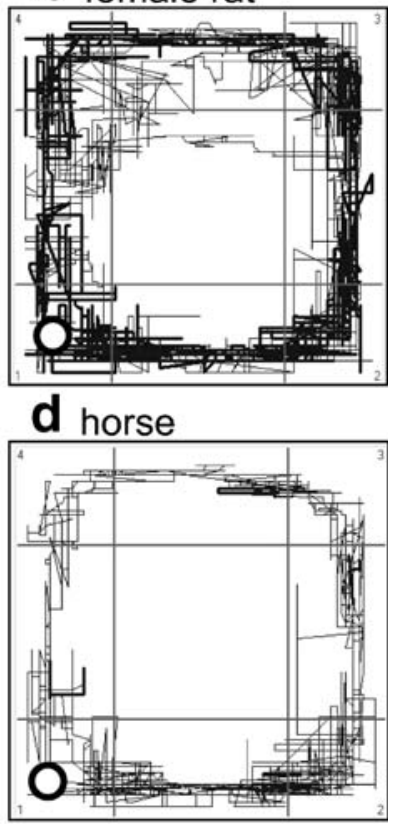

e cougar

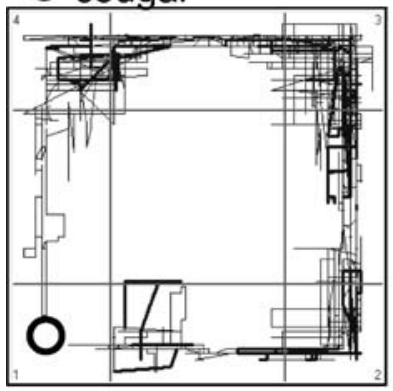

$\left.F_{3,39}=8.34, P<0.001\right)$, an increase was found in the time elapsed before contact with predator urine samples compared with that before contact with the saline controls $(t=3.60$, $P=0.003)$ and the nonpredator urine samples $(t=3.29, P=0.006)$.

For contact duration, ANOVA showed an influence of the urine samples $\left(F_{9,108}=11.41\right.$, $P<0.001$; Figure 1d). Pairwise comparison with the saline controls revealed an increase in the duration of contact with the urine of female rats $(t=3.65, P=0.004)$ and a reduced duration of contact with the urines of coyotes, wolves, and cougars $(t>3.07, P<0.01)$. However, after grouping the data (ANOVA: $F_{3,39}=14.45, P<0.001$ ), the effects of female rat urine were still found ( $t=4.21, P=0.001)$, but longer contact with nonpredator urine was found $(t=2.59, P=0.02)$. No effect of predator urine was found on contact duration relative to the saline control $(t=0.88, P=0.38)$, but there was a difference with nonpredator urine $(t=3.42, P=0.005)$.

There were no effects of individual urine samples on the rat's rearing behavior (ANOVA: $F_{9,108}=1.63, P=0.12$; Figure 1e). However, after grouping the data (ANOVA: 
$F_{3,39}=3.01, P<0.041$ ), a significant increase was found in rearing during exposure to predator urine $(t=3.32, P=0.006)$. There was also a trend to increased rearing behavior with nonpredator urine $(t=1.99, P=0.07)$. No significant difference was found in rearing behavior during exposure to predator urine and exposure to nonpredator urine $(t=1.44, P=$ $0.17)$.

Analysis of the data on urine samples both singly and as group showed no statistical effects of the predator urine samples on resting behavior, time spent in the middle of the arena, general motor activity (distance traveled), freezing, grooming, and numbers of fecal boli (data not shown).

\section{Discussion}

Our expectation was that defensive behavior is only observable during exposure to predator urine, but not during exposure to other samples. It should be noted that the rats used in the study were totally naive to all odors tested, i.e., that observed differences cannot be a consequence of some odors being unknown, with others being known. Exposure to urine samples of canid and feline predators induced different defensive behaviors in the animals. Behavioral responses to the different predator urine samples, with exception of cat urine samples, were similar. Possible reasons for this exception are discussed below.

Animals spent less time in corners with urine samples of canid and feline predators than in corners with saline controls and nonpredator urine, indicating avoidance behavior. Furthermore, flat back approaches, a form of risk assessment (Blanchard et al., 1990b), were only observed only in the presence of predator urine samples. In addition, there was a longer delay in making contact with predator urine samples, and the duration of contact with some of the predator odors (coyote, cougar, and wolf) was significantly decreased. Interestingly, rearing behavior - an indicator of exploratory behavior and vigilance - was increased in frequency during exposure to both predator and nonpredator urine. Other behavior indicators such as resting behavior, freezing, general motor activity (distance traveled), and grooming were not affected by exposure to predator urine. The first two indicators often increase and the latter two often decrease in frequency during exposure to aversive stimuli (Yamada and Nabeshima, 1995; Blanchard et al., 2003a; Misslin, 2003; Apfelbach et al., 2005). Female rat urine increased both the time spent in the sample corner and the duration of contact with the sample (cf. Markham et al., 2004), clearly showing that our experiment was sensitive not only to aversive, but also to attractive odors.

All types of behaviors analyzed in the present study are potentially modulated by danger. Freezing behavior is a species-specific defensive response in rats and is often used to measure fear (Fendt and Fanselow, 1999; Wiltgen and Fanselow, 2003; Sullivan et al., 2004). Surprisingly, freezing behavior was infrequent in the present study and was not exclusive to predator urine, ostensibly indicating that the canid and feline predator odors used here were unable to induce defensive behavior. However, this conclusion must be rejected for several reasons. Freezing behavior is observed only when the threatening stimulus is very intense, i.e., danger is acute (e.g., Blanchard and Blanchard, 1990; McNaughton and Corr, 2004). Indeed, intense stimuli such as foot shocks or high concentrations of trimethylthiazoline (a component of the fox odor) were able to induce freezing behavior in the experimental setup used in the present study (Fendt, unpublished observations). Results of the present study, therefore, indicate that the canid and feline predator urine samples used in this study are not a strong indicator of acute danger and do not induce freezing behavior. It is also questionable whether freezing would be an adequate 
response to predator odor. While predator odor indicates the presence of a predator in the area, it is not implicitly an indicator of acute and direct predatory threat. Therefore, it is more expedient for the prey animal to intensify its risk assessment behavior or to avoid an area with predator odor in order to prevent an encounter with the predator (cf., McNaughton and Corr, 2004). On the other hand, freezing responses and flight or fight behavior are more relevant when there is acute and direct danger of encountering a predator. Another possible explanation for the lack of freezing behavior in experiments involving the presence of predator odors is that the occurrence of freezing behavior depends upon the test context. It has been shown repeatedly that a threatened animal responds by freezing only if it has no way to avoid the threatening stimulus (e.g., Blanchard and Blanchard, 1971). Otherwise, it responds with flight or avoidance behavior rather than freezing behavior.

Consequently, either the quality or intensity of the stimuli in the present study or the testing context itself was such that freezing behavior and the related inhibition of spontaneous activity and of grooming were not an appropriate response for our test animals during exposure to the samples of predator urine. As discussed above, a more appropriate response when the threatening stimulus is lower in intensity might be risk assessment behavior (cf., McNaughton and Corr, 2004) such as flat back approaches (or "stretch approaches"; e.g., Markham et al., 2004). In the present study, flat back approaches were observed only in response to the urine probes of canid and feline predators and were much more frequent as the animals approached these samples.

Direct exploration of stimuli occurs much faster when the stimuli are potentially dangerous (cf., Markham et al., 2004). For example, I found that the duration of contact with the urine probes of canid and feline predators was much shorter than with other stimuli. In contrast, the urine of female rats, which is highly attractive to male rats, was explored much longer (cf., Markham et al., 2004).

Potentially dangerous stimuli are approached not only more slowly and for a shorter period of time, but they are also avoided more thereafter (reviewed in McGregor et al., 2002; Apfelbach et al., 2005; Takahashi et al., 2005). In the present study, animals spent less time in corners with urine samples of canid and feline predators than those containing urine of nonpredators and female rats or a saline control solution. Quantitatively, in fact, this type of behavior, i.e., time spent in the urine sample corner or avoidance of that corner, was the most pronounced of all the behavioral responses assessed.

In summary, different defensive behaviors were induced by urine samples of canid and feline predators, but not by the other nonpredator samples. Although the behavioral effects of the predator urine were by and large similar, there was one major exception: the cat urine did not induce defensive behavior. This has also been demonstrated by Blanchard et al. (2003b). In that study, avoidance behavior, decreased duration of contact, and increased frequency of risk assessment behavior and freezing were induced by exposure to cat feces and cat fur/skin, but not by exposure to cat urine. This may be explained as follows. First, predator urine in general appears to be less fear-inducing than the odor of feces or skin/fur (cf., Blanchard et al., 2003b; only cat odor was tested in this study). Second, cat odor may change during domestication so that its fear-inducing properties are weakened or lost altogether. Third, cat urine was acquired in the present study by bladder catheterization. Urine acquired in this way is likely to induce less fear, as the urine used for scent marking is usually enriched by secretions of the anal gland. Fourth, domesticated cats may have a vegetarian or mixed diet changing the properties of the cat odor especially of the urine odor (cf., Berton et al., 1998).

In contrast to cat urine, urine of the other canid and feline predators used in the present study induced defensive behavior. Similar effects have been shown in a number of studies 
with other prey species: beavers, black-tailed deer, snowshoe hares, deer mice, guinea pigs, house mice, gray squirrels, meadow voles, and mountain voles during exposure to coyote, wolf, fox, and bobcat urine (Sullivan et al., 1985a,b, 1988; Epple et al., 1993; Nolte et al., 1994; Swihart et al., 1999; Rosell, 2001), and, most relevantly, in rats during exposure to fox urine (Burwash et al., 1998; Campbell et al., 2003; Farmer-Dougan et al., 2005). The results of all these studies were confirmed here. In contrast to the previous work, however, the present study used (1) both predator and nonpredator urine samples and (2) laboratory animals with no experience whatever of predators or their odors. In other words, this present experiment is the first to conclusively demonstrate that several predator odors (here: canid and feline urine), but not other natural odors (here: urine of conspecifics and herbivores) induce innate defensive behavior in inexperienced rats.

Taken as a whole, the study showed that exposure to canid and feline predator urine induces defensive behaviors, particularly avoidance and risk assessment behavior, in predator-odor naive rats. It should be mentioned that this study is limited in that only canid and feline urine was used as predator odors and only herbivore urine was used as nonpredator odor. However, it is the first to test different predator and nonpredator probes in laboratory-reared animals. The results support the hypothesis that the effect of predator odors on the behavior of many species observed in numerous studies (reviewed in Kats and Dill, 1998; Apfelbach et al., 2005) is based on an innate recognition of predator odors. Such an innate "recognition" of predator odor is a survival advantage. In parallel, an innate recognition of visual and acoustic predator cues has also been discussed in other studies (e.g., Hirsch and Bolles, 1980; Hendrie, 1991; Hendrie et al., 1996; Eilam et al., 1999).

Acknowledgments The author thanks Daniel Endler, Daniela Schmieder, and Verena Koller for assistance with these experiments, as well as Dr. Björn Siemers for comments on the manuscript.

\section{References}

AMO, L., LÓPEZ, P. and MARTín, J. 2004. Trade-offs in the choice of refuges by common wall lizards: Do thermal costs affect preferences for predator-free refuges? Can. J. Zool. 82:897-901.

Apfelbach, R., Blanchard, C. D., Blanchard, R. J., Hayes, R. A. and Mcgregor, I. S. 2005. The effects of predator odors in mammalian prey species: A review of field and laboratory studies. Neurosci. Biobehav. Rev. 29:1123-1144.

Berton, F., Vogel, E. and Belzung, C. 1998. Modulation of mice anxiety in response to cat odor as a consequence of predators diet. Physiol. Behav. 65:247-254.

Blanchard, R. J. and Blanchard, D. C. 1971. Defensive reactions in the albino rat. Learn. Motiv. 2:351362.

Blanchard, R. J. and Blanchard, D. C. 1990. Anti-predator defense as models of animal fear and anxiety, pp. 89-108, in P. F. Brain, S. Parmigiani, and R. J. Blanchard (eds.). Fear and Defence. Church and Harwood Academic, New York.

Blanchard, R. J., Blanchard, D. C., Rodgers, J., and Weiss, S. M. 1990a. The characterization and modeling of antipredator defensive behavior. Neurosci. Biobehav. Rev. 14:463-472.

Blanchard, R. J., Blanchard, D. C., Weiss, S. M., and Meyer, S. 1990b. The effects of ethanol and diazepam on reactions to predatory odors. Pharmacol. Biochem. Behav. 35:775-780.

Blanchard, D. C., Griebel, G., and Blanchard, R. J. 2003a. The Mouse Defense Test Battery: Pharmacological and behavioral assays for anxiety and panic. Eur. J. Pharmacol. 463:97-116.

Blanchard, D. C., Markham, C., Yang, M., Hubbard, D., Madarang, E., and Blanchard, R. J. 2003 b. Failure to produce conditioning with low-dose trimethylthiazoline or cat feces as unconditioned stimuli. Behav. Neurosci. 117:360-368.

Burwash, M. D., Tobin, M. E., Woolhouse, A. D., and Sullivan, T. P. 1998. Laboratory evaluation of predator odors for eliciting an avoidance response in roof rats (Rattus rattus). J. Chem. Ecol. 24: 49-65. 
Campbell, T., Lin, S., Devries, C., and Lambert, K. 2003. Coping strategies in male and female rats exposed to multiple stressors. Physiol. Behav. 78:495-504.

Carobrez, A. P., Teixeira, K. V., and Graeff, F. G. 2001. Modulation of defensive behavior by periaqueductal gray NMDA/glycine-B receptor. Neurosci. Biobehav. Rev. 25:697-709.

DiCKMAN, C. R. 1992. Predation and habitat shift in the house mouse, Mus domesticus. Ecology 73: 313-322.

Dickman, C. R. and Doncaster, C. P. 1984. Responses of small mammals to red fox (Vulpes vulpes) odour. J. Zool. 204:521-531.

Dielenberg, R. A. and MCGregor, I. S. 2001. Defensive behavior in rats towards predatory odors: A review. Neurosci. Biobehav. Rev. 25:597-609.

EDUT, S. and EILAM, D. 2003. Rodents in open space adjust their behavioral response to the different risk levels during barn-owl attack. BMC Ecol. 3:10-22.

Eilam, D., Dayan, T., Ben-Eliyahu, S., Schulman, I. I., Shefer, G., and Hendrie, C. A. 1999. Differential behavioural and hormonal responses of voles and spiny mice to owl calls. Anim. Behav. 58:1085-1093.

Epple, G., Mason, J. R., Nolte, D. L., and Campbell, D. L. 1993. Effects of predator odors on feeding in the mountain beaver (Aplodontia rufa). J. Mammol. 74:715-722.

Farmer-Dougan, V., Chandrashekar, S., Stutzman, D., Bradham, K., and Dougan, J. D. 2005. Fox urine as an aversive stimulus: Modification of a passive avoidance task. J. Gen. Psychol. 132:313-320.

FENDT, M. and FANSELOW, M. S. 1999. The neuroanatomical and neurochemical basis of conditioned fear. Neurosci. Biobehav. Rev. 23:743-760.

Fendt, M., Endres, T., Lowry, C. A., Apfelbach, R., and McGregor, I. S. 2005. TMT-induced autonomic and behavioral changes and the neural basis of its processing. Neurosci. Biobehav. Rev. 29:1145-1156.

GeSE, E. M. and RufF, R. L. 1997. Scent-marking by coyotes, Canis latrans: The influence of social and ecological factors. Anim Behav. 54:1155-1166.

GlowacinsKi, Z. and Profus, P. 1997. Potential impact of wolves Canis lupus on prey populations in eastern Poland. Biol. Cons. 80:99-106.

Goldyn, B., Hromada, M., Surmacki, A., and Tryjanowski, P. 2003. Habitat use and diet of the red fox Vulpes vulpes in an agricultural landscape in Poland. Z. Jagdwiss. 49:191-200.

HENDRIE, C. A. 1991. The calls of murine predators activate endogenous analgesia mechanisms in laboratory mice. Physiol. Behav. 49:569-573.

Hendrie, C. A., Weiss, C., and EILAM, D. 1996. Exploration and predation models of anxiety: Evidence from laboratory and wild species. Pharmacol. Biochem. Behav. 54:13-20.

HiRsCH, S. M. and Bolles, R. C. 1980. On the ability of prey to recognize predators. Z. Tierpsychol. 54: 7184.

KATS, L. B. and DiLL, L. M. 1998. The scent of death: Chemosensory assessment of predation risk by prey animals. Ecoscience 5:361-394.

LABRA, A. and NiEMEYER, H. M. 2004. Variability in the assessment of snake predation risk by Liolaemus lizards. Ethology 110:649-662.

Markham, C. M., Blanchard, D. C., Canteras, N. S., Cuyno, C. D., and Blanchard, R. J. 2004. Modulation of predatory odor processing following lesions to the dorsal premammillary nucleus. Neurosci. Lett. 372:22-26.

McGregor, I. S., Schrama, L., Ambernoon, P., and Dielenberg, R. A. 2002. Not all 'predator odours' are equal: Cat odour but not 2,4,5 trimethylthiazoline (TMT; fox odour) elicits specific defensive behaviours in rats. Behav. Brain Res. 129:1-16.

MCNAughton, N. and CORR, P. J. 2004. A two-dimensional neuropsychology of defense: Fear/anxiety and defensive distance. Neurosci. Biobehav. Rev. 28:285-305.

Misslin, R. 2003. The defense system of fear: Behavior and neurocircuitry. Neurophysiol. Clin. 33:55-66.

Nolte, D. L., Mason, J. R., Epple, G., Aronov, E., and Campbell, D. L. 1994. Why are predator urines aversive to prey? J. Chem. Ecol. 20:1505-1516.

Rosell, F. 2001. Effectiveness of predator odors as grey squirrel repellents. Can. J. Zool. 79:1719-1723.

STAPLEY, J. 2003. Differential avoidance of snake odours by a lizard: Evidence for prioritized avoidance based on risk. Ethology 109:785-796.

Sullivan, T. P., Nordstrom, L., and Sullivan, D. S. 1985a. Use of predator odors as repellents to reduce feeding damage by herbivores. I. Showshoe hares (Lepus americanus). J. Chem. Ecol. 11:903-911.

Sullivan, T. P., Nordstrom, L., and Sullivan, D. S. 1985b. Use of predator odors as repellents to reduce feeding damage by herbivores. II. Black-tailed deer (Odocoileus hemionus columbianus). J. Chem. Ecol. 11:921-935. 
Sullivan, T. P., CrumP, D., and Sullivan, D. S. 1988. Use of predator odors as repellents to reduce feeding damage by herbivores. III. Montane and meadow voles (Microtus montanus and Microtus pennsylvannicus). J. Chem. Ecol. 14:363-378.

Sullivan, G. M., Apergis, J., Bush, D. E., Johnson, L. R., Hou, M., and Ledoux, J. E. 2004. Lesions in the bed nucleus of the stria terminalis disrupt corticosterone and freezing responses elicited by a contextual but not by a specific cue-conditioned fear stimulus. Neuroscience 128:7-14.

Swihart, R. K., Pignatello, J. J., and MattinA, M. J. I. 1999. Adverse responses of white-tailed deer, Odocoileus virginianus, to predator urines. J. Chem. Ecol. 17:767-777.

Takahashi, L. K., Nakashima, B. R., Hong, H., and Watanabe, K. 2005. The smell of danger: A behavioral and neural analysis of predator odor-induced fear. Neurosci. Biobehav. Rev. 29:1157-1167.

WiLtgen, B. J. and FANSELOW, M. S. 2003. A model of hippocampal-cortical-amygdala interactions based on contextual fear conditioning, pp. 83-103, in K. J. Jeffries (ed.) The Neurobiology of Spatial Behavior. Oxford University Press, Oxford.

YAMADA, K. and NABESHIMA, T. 1995. Stress-induced behavioral responses and multiple opioid systems in the brain. Behav. Brain Res. 67:133-145.

ZaR, J. H. 1999. Biostatistical Analysis. Prentice-Hall, Upper Saddle River, NJ. 